\title{
From Catastrophizing to Recovery: a pilot study of a single-session treatment for pain catastrophizing
}

This article was published in the following Dove Press journal: Journal of Pain Research

25 April 2014

Number of times this article has been viewed

\section{Beth D Darnall \\ John A Sturgeon \\ Ming-Chih Kao \\ Jennifer M Hah \\ Sean C Mackey}

Division of Pain Medicine, Stanford Systems Neuroscience and Pain Laboratory, Stanford University School of Medicine, Palo Alto, CA, USA
Correspondence: Beth D Darnall Division of Pain Medicine, Stanford Systems Neuroscience and Pain Laboratory, Stanford University School of Medicine, 1070 Arastradero, Suite 200, MC 5596 Palo Alto, CA 94304-1336, USA

Email bdarnall@stanford.edu
Background: Pain catastrophizing (PC) - a pattern of negative cognitive-emotional responses to real or anticipated pain - maintains chronic pain and undermines medical treatments. Standard PC treatment involves multiple sessions of cognitive behavioral therapy. To provide efficient treatment, we developed a single-session, 2-hour class that solely treats PC entitled "From Catastrophizing to Recovery" [FCR].

Objectives: To determine 1) feasibility of FCR; 2) participant ratings for acceptability, understandability, satisfaction, and likelihood to use the information learned; and 3) preliminary efficacy of FCR for reducing PC.

Design and methods: Uncontrolled prospective pilot trial with a retrospective chart and database review component. Seventy-six patients receiving care at an outpatient pain clinic (the Stanford Pain Management Center) attended the class as free treatment and 70 attendees completed and returned an anonymous survey immediately post-class. The Pain Catastrophizing Scale (PCS) was administered at class check-in (baseline) and at 2, and 4 weeks post-treatment. Within subjects repeated measures analysis of variance (ANOVA) with Student's $t$-test contrasts were used to compare scores across time points.

Results: All attendees who completed a baseline PCS were included as study participants $(\mathrm{N}=57 ; F=82 \%$; mean age $=50.2$ years); PCS was completed by 46 participants at week 2 and 35 participants at week 4 . Participants had significantly reduced $\mathrm{PC}$ at both time points $(P<0001)$ and large effect sizes were found (Cohen's $d=0.85$ and $d=1.15$ ).

Conclusion: Preliminary data suggest that FCR is an acceptable and effective treatment for PC. Larger, controlled studies of longer duration are needed to determine durability of response, factors contributing to response, and the impact on pain, function and quality of life.

Keywords: chronic pain, cognitive behavioral therapy, pain treatment, chronic debilitation, pain treatment costs, pain psychology

\section{Introduction}

Chronic pain exerts an enormous impact on the quality of life of the more than 100 million people in the US ${ }^{1}$ and more than 1 billion people around the globe who are living with this condition. ${ }^{2}$ Moreover, the economic burden of chronic pain is staggering: in the US alone the combined annual costs of treatment and related disability exceed half a trillion dollars. Accordingly, there is an urgent need to treat the factors that maintain and worsen the pain experience in order to reduce human suffering, pain treatment costs, and chronic debilitation.

Pain catastrophizing (PC) is foremost among the psychological factors that worsen pain and contribute to pain chronicity. PC is defined as persistent negative cognitive 
and emotional responses to actual or anticipated pain. ${ }^{3}$ In part, PC contributes to a cycle of futility in the medical treatment realm because it undermines behavioral and medical treatments. $^{4-11}$

Across chronic pain conditions, PC has been associated with an array of negative phenomena including greater pain intensity, ${ }^{12-17}$ affective distress, ${ }^{8}$ muscle and joint tenderness, ${ }^{13}$ muscular tension at rest, ${ }^{18}$ pain-related disability, ${ }^{13,14,16,19-21}$ poor response to various pain treatments ${ }^{8,18,22}$ including surgery, ${ }^{16,23,38}$ and to greater use ${ }^{23}$ and misuse of opioids. ${ }^{24}$

$\mathrm{PC}$ is also harmful in the context of acute pain, and even for individuals who are pain free because PC is associated with an increased risk for developing chronic pain. For instance, $\mathrm{PC}$ is implicated in the persistence of back pain ${ }^{21}$ and it has been shown to account for $47 \%$ of the variance in the development of chronic back pain following an episode of acute back pain. ${ }^{25}$ Moreover, a population study revealed that among all factors investigated, PC best predicted the acquisition of chronic low back pain 1 year after a pain free baseline. ${ }^{26}$ In combination, data from multiple studies ${ }^{4-26,38}$ show that PC is detrimental, and that early treatment for PC may serve as chronic pain prophylaxis. ${ }^{21,25,26}$

Given that PC clearly undermines response to medical treatment for chronic pain it is perhaps unsurprising that reductions in PC are associated with subsequent salutary effects for pain and interventions. In a prospective treatment outcome study, Burns et al used cross-lagged analysis to show that early reductions in PC led to subsequent improved response to multidisciplinary chronic pain treatment as evidenced by significant reductions in pain intensity and pain interference. ${ }^{27}$ Similar findings were reported for a study of women with fibromyalgia, with early reductions in PC predicting later improvement in fibromyalgia pain. ${ }^{28}$ Considered collectively, these studies underscore the need to improve access to PC treatment in order to reduce pain and to optimize response to other treatments. Indeed, some models suggest that $\mathrm{PC}$ is a normative response to pain albeit maladaptive - rather than an idiosyncratic and deviant phenomenon, ${ }^{29}$ thus providing additional, broad rationale for the need and applicability for specific PC treatment.

$\mathrm{PC}$ is primarily treated with cognitive behavioral therapy (pain-CBT) $^{30}$ delivered across multiple individual psychotherapy sessions or in pain coping skills classes that typically span 6-10 sessions and cover many topics in addition to PC (eg, pacing, communication skills, daily activity and exercise, and sleep hygiene). ${ }^{16,31}$ As such, the burdens of time, travel, and cost associated with multiple visits to a psychologist may stand as barriers to care, thus leaving PC untreated.
While observing the need to improve treatment efficiency, we found no reports of a single-session treatment that solely targets PC.

Accordingly, we developed and pilot tested a singlesession, 2-hour pain-CBT class for PC entitled: "From Catastrophizing to Recovery" (FCR). This study of FCR had several goals: 1) to determine feasibility in a mixedetiology chronic pain clinic sample; 2) to acquire pilot data regarding anonymous participant ratings for FCR in terms of acceptability, satisfaction with the intervention, understandability of the information presented, and likelihood for using the information and skills learned in the class; and 3) to determine preliminary efficacy as measured by changes in participants' PC scores over time (scores at class check-in [baseline] were compared to scores post-treatment at followup 2, and 4 weeks after class attendance).

We hypothesized good feasibility for the FCR intervention in patients receiving treatment at a chronic pain clinic. We also hypothesized high mean participant ratings ( $\geq 80 \%)$ for the anonymous post-class survey in terms of treatment acceptability, participant satisfaction, understandability of the information, usefulness, and likelihood to use the information and skills learned. Finally, we hypothesized that FCR participants would have significantly reduced PC at 2, and 4 weeks post-treatment.

\section{Design and methods}

The study design was an uncontrolled prospective pilot trial with a retrospective chart review component. Data sources included a self-report measure, the Pain Catastrophizing Scale $(\mathrm{PCS})^{3}$ administered at three time points, (pre-treatment and at 2 , and 4 weeks post-treatment) and medical chart review (determining age, sex, and current anxiety and depression diagnoses). The study was conducted in compliance with the Stanford Institutional Review Board. Participants comprised patients receiving care at the Stanford Pain Management Center, an academic, multidisciplinary chronic pain clinic.

Participants were referred to the class by one of their treatment providers (ie, a pain psychologist, registered nurse, physician assistant, physical therapist or pain physician) for free specialty pain treatment. At the point of referral, participants received a class flyer that included class logistics in terms of time, date, location, and the purpose of the class (acquiring mind-body information and skills to gain control over catastrophizing). While clinic providers tended to refer patients they felt could benefit from a skills-building class to combat catastrophizing tendencies, no specific eligibility criteria or screening procedures were 
used. No compensation was offered for participating in the class or in the follow-up component.

\section{Measures}

\section{Pain catastrophizing scale}

The $\mathrm{PCS}^{3}$ is a 13-item self-report questionnaire widely used to assess catastrophizing tendencies in chronic pain research and clinical settings. The PCS directs respondents to consider how they tend to think and feel in the broad context of pain stimuli. A sample item from the PCS: "I become afraid that the pain will get worse". Respondents rate their endorsement of frequency for each item using a $0-4$ Likert scale $(0=$ not at all; $4=$ all the time). The PCS is comprised of three subscales: magnification, rumination, and feelings of helplessness. All items are summed to create a total score. The psychometric sufficiency of the PCS has been demonstrated. ${ }^{3,32-34}$ The PCS was administered at class check-in (baseline) and posttreatment (2, and 4 weeks after class attendance) to determine further treatment needs (participants who evidenced negligible treatment response were referred for individual psychotherapy). At 2 , and 4 weeks post-treatment participants received an email with a link to the PCS (housed within the Stanford Research Electronic Data Capture [REDCap]). ${ }^{35}$ Once a participant completes their PCS data entry the form is immediately locked and cannot be edited.

\section{Anonymous post-class survey}

The post-class survey was administered immediately after each FCR class. Participants did not provide identifying information and the surveys were returned in a group folder to further ensure anonymity. The surveys contained the following five questions: 1) How acceptable did you find this class to be? 2) How satisfied are you with this class? 3) How useful was the information presented? 4) Was the information easy to understand? 5) How likely are you to use the skills and information you learned today? Responses were rated on a 7-point Likert scale that was tailored to match each question (eg, How likely are you to use the skills and information you learned today? Response set: $0=$ not at all likely, $6=$ highly likely).

\section{Chart review}

Electronic medical charts were reviewed to collect information regarding participant age, sex, and psychological diagnoses for current anxiety or major depressive disorder. We did not extract information regarding pain diagnoses as the majority of patients receiving care at the pain clinic have multiple comorbid pain conditions.

\section{Treatment intervention FCR class}

Delivery: FCR was delivered by a licensed clinical psychologist via a PowerPoint presentation to groups of patients in a single session lasting 130 minutes.

Content: FCR has two main components: didactics and skills acquisition. Didactic content includes mind-body science as it relates to pain and PC. Participants learn how to identify catastrophizing in the moment, and how to self-treat it. During the class, participants acquire skills and develop a plan to apply the learned skills to decrease physiological hyperarousal - diaphragmatic breathing and progressive muscle relaxation - within the context of PC. Participants also acquire skills that improve the regulation of cognition and emotion, including PC reframing and thought restructuring, and develop a plan for implementing these skills in daily life. During the class participants identify their typical PC thoughts and practice writing out their reframes. Finally, participants develop a plan to use behaviors that modulate attention and counteract helplessness. During the class, participants create personalized lists of self-soothing behaviors used to stop PC; lists are customized to various settings. Participants self-tailor the information relayed during the class by developing their own comprehensive self-treatment plan to stop and prevent catastrophizing. Participants leave the class with the following tangibles: 1) their self-written, self-crafted, personalized PC cessation plan; 2) a 20-minute relaxation response audio $\mathrm{CD}$; and 3) a printed copy of the FCR content to access as needed in their PC cessation plan.

\section{Analyses}

Descriptive statistics included means and standard deviations (SDs) for demographics. Anxiety and depression diagnoses were coded as binary variables. Means, SDs, and percentage ratings were calculated for the 5-item anonymous post-class surveys. Total scores were calculated for the PCS at each time point (baseline, and 2, and 4 weeks post-treatment). Treatment response was quantified for each subject using percentage change from baseline PCS to each follow-up time point. The clinical importance of the change in PCS scores at both follow up time points was then categorized in accordance with Initiative on Methods, Measurement, and Pain Assessment in Clinical Trials (IMMPACT) recommendations: $<15 \%$ change in the primary outcome was clinically unimportant, a $15 \%-30 \%$ change was minimally clinically important, a $30 \%-50 \%$ change was moderately clinically important, and $\geq 50 \%$ change was substantially clinically important. ${ }^{36}$ 
Using SAS Enterprise Guide 6.1 (SAS Institute Inc., Cary, NC, USA), repeated measures ANOVA was used to account for subject variability using PROC GLM. Data were examined for percentage of missing PCS scores. Imputation was completed with last observation carried forward of the PCS score. Resulting models were examined to ensure sphericity. In addition, the variance and covariance matrices for PCS scores were examined at all three time points to assess for compound symmetry. Student's paired $t$-tests were used to compare PCS scores between time points. Cohen's $d$ was used to determine treatment effect sizes.

\section{Results}

\section{Sample and demographics}

Seventy-six patients with chronic pain of mixed pain etiology and diagnoses attended FCR across 13 class cohorts between June and December 2013. Class cohort size ranged between 4-18 participants. Of the 76 attendees, 57 completed and returned a baseline PCS during the class and thus comprised our study sample. The study sample was predominantly female (71.9\%) with a mean age of 50.2 years $(\mathrm{SD}=12.2$; range $18-69$ years). Chart review indicated that $47.4 \%(\mathrm{~N}=27)$ of the 57 participants with at least one PCS score had a diagnosis of anxiety and $42.1 \%(\mathrm{~N}=24)$ had a diagnosis of depression.

\section{Anonymous post-class survey}

Mean ratings exceeded $90 \%$ for each question contained in the anonymous post-class survey (Table 1), and thus exceeded our a priori threshold of $\geq 80 \%$ for each item. Because the surveys were anonymous we were unable to distinguish surveys that were linked to study participants versus those linked to non-participants (ie, class attendees who did not complete a baseline PCS); as such, the data presented

Table I Post-class survey results $(\mathrm{N}=70)$

\begin{tabular}{lll}
\hline Item & $\begin{array}{l}\text { Mean } \\
\text { (SD) }\end{array}$ & $\begin{array}{l}\text { Percentage above } \geq \mathbf{8 0} \% \\
\text { threshold }\end{array}$ \\
\hline $\begin{array}{l}\text { How acceptable did you } \\
\text { find this class? }\end{array}$ & $5.6(0.67)$ & Acceptability: 93\% \\
$\begin{array}{l}\text { How satisfied are you } \\
\text { with this class? }\end{array}$ & $5.5(0.75)$ & Satisfaction: 92\% \\
$\begin{array}{l}\text { How useful was the } \\
\text { information presented? }\end{array}$ & $5.6(0.67)$ & Usefulness: 94\% \\
$\begin{array}{l}\text { Was the information } \\
\text { easy to understand? } \\
\text { How likely are you to use } \\
\text { the skills and information } \\
\text { you learned today? }\end{array}$ & $5.5(0.60)$ & Ease of understanding: 95\% \\
\hline
\end{tabular}

Abbreviation: SD, standard deviation. in Table $1(\mathrm{~N}=70)$ included class attendees who did not participate in the PCS follow-up component.

Week 2 data were missing for eleven of the 57 study participants (19.2\%), and week 4 data were missing for 22 participants (38.6\%). All 57 participants reported at least one follow-up PCS score at either week 2 or week 4 . Thus, only $28.9 \%$ of data regarding the PCS scores were missing, which is considered acceptable. ${ }^{37}$ Thus, the decision was made to proceed with imputation using last observation carried forward.

\section{PCS means}

PCS means and SDs for each time point are presented in Table 2. The PCS was found to be normally distributed at baseline, and non-normality tests were non-significant. At both week 2 and week 4 post-treatment, the PCS was skewed toward lower scores with significant non-normality tests at each time point.

\section{ANOVA}

Repeated measures ANOVA (rANOVA) was used to account for subject variability. Results revealed a significant linear effect for time indicating substantially reduced PCS scores at weeks 2 and 4 compared to baseline $(F[2,112]=52.5$, $P<0.0001)$. Post hoc paired $t$-tests revealed significant within subjects differences for all contrasts: PCS baseline to week 2 ( $t=7.01, P<0.001)$; PCS baseline to week $4(t=8.36$, $P<0.001)$; and PCS week 2 to week 4 ( $t=2.51, P=0.02)$. Sphericity was maintained. In addition, the variance and covariance matrices of the PCS endpoint were examined to ensure compound symmetry for univariate analysis. When examining an rANOVA model comparing those with baseline depression to those without baseline depression, response profiles did not differ significantly by depression status based on the interaction between time and depression status $(F[2,74]=2.26, P=0.11)$ (Figure 1$)$. However, a significant overall difference was found between subjects $(F[1,37]=5.82, P=0.02)$ for the reported PCS scores, such that participants with baseline depression tended to report significantly higher scores on the PCS across time points than participants without baseline depression. The overall effect

Table 2 PCS means by time point $(\mathrm{N}=57)$

\begin{tabular}{ll}
\hline Time point & Mean (SD) \\
\hline Baseline & $26.1(10.8)$ \\
Post-treatment week 2 & $16.5(9.9)$ \\
Post-treatment week 4 & $13.8(9.5)$ \\
\hline
\end{tabular}

Abbreviations: PCS, Pain Catastrophizing Scale; SD, standard deviation. 


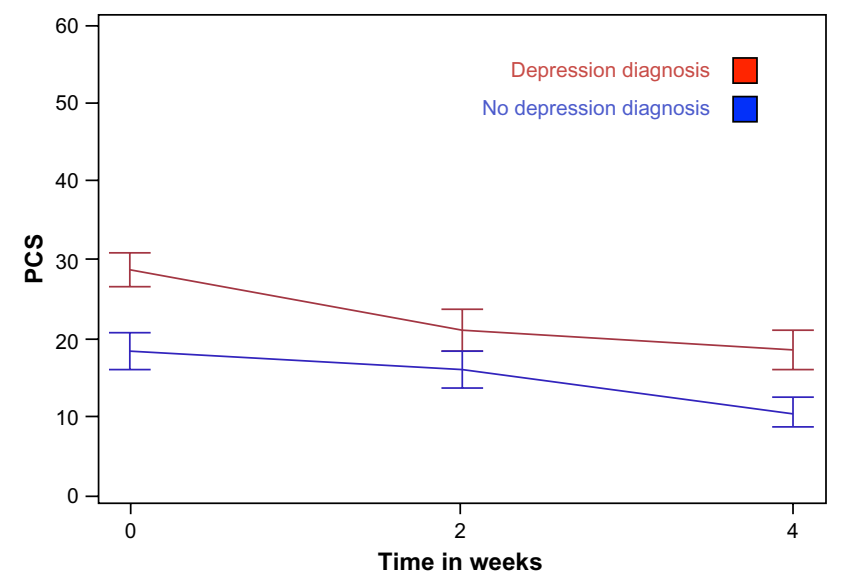

Figure I PCS means by time point for depression diagnosis (yes/no). Abbreviation: PCS, Pain Catastrophizing Scale.

of time within subjects remained significant in this model $(F[2,74]=24.45, P<0.0001)$.

Similarly, when examining an rANOVA model comparing those with baseline anxiety to those without baseline anxiety (Figure 2), response profiles did not differ significantly by anxiety status based on the interaction between time and anxiety status $(F[2,74]=0.22, P=0.80)$. However, a significant overall difference was found between subjects $(F[1,37]=7.82$, $P=0.0081$ ) for the reported PCS scores, such that participants with baseline anxiety tended to report significantly higher scores on the PCS across time points than participants without baseline anxiety. The overall effect of time within subjects remained significant in this $\operatorname{model}(F[2,74]=21.29$, $P<0.0001)$.

Finally, an rANOVA model was examined including both baseline depression and anxiety as predictors. Change in the PCS score based on the interaction of baseline anxiety or depression with time was not significant within all subjects

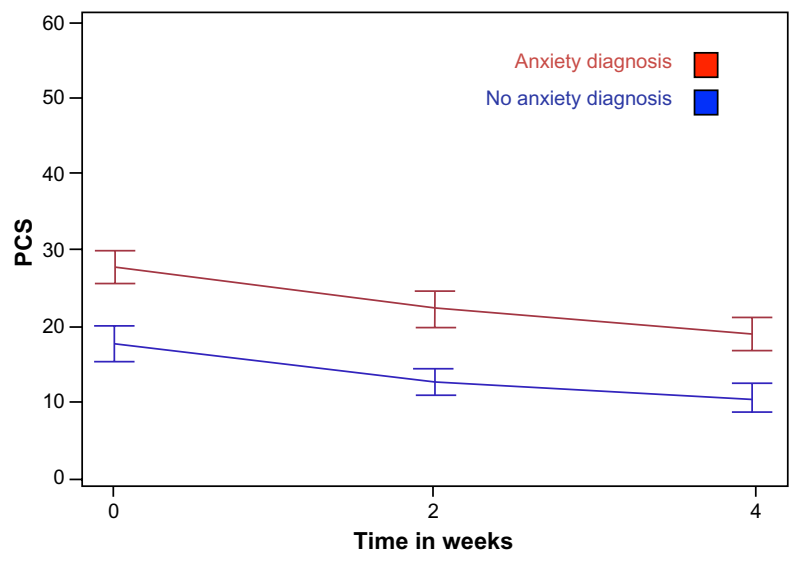

Figure 2 PCS means by time point for anxiety diagnosis (yes/no). Abbreviation: PCS, Pain Catastrophizing Scale.
$(F[2,64]=4.19, P=0.05$ and $F[2,64]=2.51, P=0.12$, respectively). Change in the PCS score over time within all subjects remained significant $(F[2,64]=18.54, P<0.0001)$. However, PCS scores did not vary significantly between participants with and without baseline anxiety or depression in this model $(F[2,64]=0.37, P=0.69$ and $F[2,64]=2.46, P=0.09$ respectively).

\section{Effect sizes}

Treatment effect sizes were large for baseline to week 2 post-treatment (Cohen's $d=0.85$ ) and for baseline to week 4 post-treatment (Cohen's $d=1.15$ ).

\section{Clinical importance}

We evaluated treatment response for participants. Table 3 displays the clinical importance of PCS changes and the number of participants in each category of clinical importance by follow-up time point based on the analytic dataset with imputed values.

Overall, 51 participants (91\% of those with followup data) achieved reductions in PC that are considered clinically important either at week 2 or week 4 , with $66.7 \%$ $(\mathrm{N}=38)$ achieving moderately or substantially important reductions. If we conservatively estimate that everyone who did not participate in the follow-up component had no significant treatment response, the clinically important responder rate $(\mathrm{N}=51)$ would represent $67.1 \%$ of the original sample of 76 patients who attended FCR. The baseline PCS of patients responding to FCR at any time (either week 2 or week 4) with at least a minimally important change was 26.5 (10.0). In contrast, the baseline PCS of patients who did not respond to FCR at any time with at least a minimally important change was 23.3 (17.0). Thus, there was no difference in baseline PCS scores between these groups $(t=-0.71, P=0.48)$.

Table 3 Clinical importance of post-intervention PCS changes

\begin{tabular}{|c|c|c|c|c|}
\hline \multirow[t]{2}{*}{ Clinical importance } & \multicolumn{2}{|c|}{ Week 2} & \multicolumn{2}{|c|}{ Week 4} \\
\hline & $\mathbf{N}$ & $\begin{array}{l}\text { PCS change } \\
\text { from baseline }\end{array}$ & $\mathbf{N}$ & $\begin{array}{l}\text { PCS change } \\
\text { from baseline }\end{array}$ \\
\hline Increased PCS & 5 & $+19.8(21.6) \%$ & 3 & $+4 \mathrm{I} .3(2 \mathrm{I} .6) \%$ \\
\hline No change $(<15 \%)$ & 15 & $-1.2(2.8) \%$ & 5 & $-6.5(2.7) \%$ \\
\hline $\begin{array}{l}\text { Minimally important } \\
(15 \%-30 \%)\end{array}$ & 10 & $-23.3(3.2) \%$ & 12 & $-22.4(4.5) \%$ \\
\hline $\begin{array}{l}\text { Moderately important } \\
(30 \%-50 \%)\end{array}$ & 13 & $-40.6(4.9) \%$ & 16 & $-38.4(4.7) \%$ \\
\hline $\begin{array}{l}\text { Substantially important } \\
(\geq 50 \%)\end{array}$ & 14 & $-61.3(11.9) \%$ & 21 & $-67.2(12.3) \%$ \\
\hline
\end{tabular}

Abbreviation: PCS, Pain Catastrophizing Scale. 


\section{Discussion}

This pilot study tested the feasibility and preliminary efficacy of a single-session PC treatment in a mixed sample of 57 chronic pain patients from a multidisciplinary pain clinic. Feasibility was demonstrated in terms of patient enrollment and participation in the class, successful delivery of the group intervention across 13 class cohorts, collection of pre-post treatment data, and collection of longitudinal outcomes using an electronic system (REDCap).

The highly positive results for the anonymous post-class survey suggest that FCR was well-received among participants, and that the majority of participants felt the class offered valuable information that they anticipated integrating into their lives.

Our finding of significantly reduced $\mathrm{PC}$ at 2 , and 4 weeks post-treatment for those who completed a baseline PCS $(\mathrm{N}=57$; both $P$-values $<0.001)$ signal preliminary efficacy for our single-session intervention. We also found large effect sizes for both follow-up time points (Cohen's $d=0.85$ for week 2, and $d=1.15$ for week 4 ). Results suggest that the positive treatment effect may strengthen over time, as indicated by the increasing effect sizes. Additionally, the available data from participants who completed both follow-up assessments suggest that PCS continues to decrease throughout 4 weeks after treatment. These findings are particularly promising given that the class is delivered in a single session and participants have no ongoing therapist contact. It is unclear whether the strengthening effect may be mediated by daily or regular use of cognitive/behavioral/ physiological regulation skills, and this is highlighted as an area of importance for future studies that investigate mechanisms of treatment.

Our finding that a single-session class may effectively reduce $\mathrm{PC}$ is particularly exciting because this novel form of specific and concentrated pain-CBT treatment may greatly expand access to low-cost, efficient care. Current treatment for PC involves multiple visits to a psychologist and therefore imposes substantial burden on patients in terms of time, travel, and cost. Such burdens may stand as barriers to care, thus leaving many patients without treatment for PC. As one illustration of this point, a primary reason people with chronic pain cite when declining to participate in studies that involve group pain-CBT is the amount of time required to attend up to ten group treatment sessions. ${ }^{31}$

Previous studies have shown that early reductions in PC are associated with subsequent improved response to other pain treatment modalities. ${ }^{27,28}$ Accordingly, there may be specific value in administering the FCR treatment as early as possible in the pain treatment process; future studies may examine whether the brief intervention optimizes response to multidisciplinary pain treatment or specific modalities, such as physical therapy.

Participants diagnosed with an anxiety or depressive disorder had higher baseline PCS scores, and this is consistent with the broader literature. ${ }^{12,39,40}$ Participants with baseline depression and anxiety diagnoses started and ended the study with higher PCS scores, but within-subjects' treatment response remained similar to those without these diagnoses. These findings suggest that the brief catastrophizing treatment is effective, even for those who have been diagnosed with anxiety or depression. Regardless, we acknowledge that our relatively small sample size points to the need to better characterize the role of anxiety and depressive disorders in adherence and longitudinal outcomes.

Our sample was predominantly female, and therefore we lacked sufficient balance to test sex differences, though we highlight this as an important consideration for future research.

Strengths of this pilot study include the repeated measures and within-subjects design. The sample included a general chronic pain clinic population, with a broad range of catastrophizing tendencies, and with the typical psychological comorbidities of anxiety and depression. Accordingly, while preliminary, our data suggest that the intervention may be broadly well-suited for diverse chronic pain populations.

Several limitations merit consideration. The main limitation of this pilot study is the uncontrolled design, which does not allow us to parse out any nonspecific treatment effects of the class environment or the process by which participants were referred to the class. However, the single-session format of the FCR intervention, combined with minimal participant interaction, would likely attenuate any nonspecific effects. Nevertheless, randomized controlled studies are needed to characterize responders and non-responders to such a singlesession intervention that entirely relies on self-application of skills in the absence of ongoing therapist contact. Future studies may determine which patients are most suitable for a brief catastrophizing intervention and which patients require a high level of care.

A second limitation is the fact that our study sample comprised the 57 participants who returned a baseline PCS during the class. We were unable to track the 19 attendees who did not return a baseline PCS and therefore it is possible that these 19 attendees would evidence a reduced response to the class and their absence from the dataset would lead to an overestimation of treatment effects. 
Of our 57 participants, $10.5 \%(\mathrm{~N}=6)$ did not complete a follow-up PCS. Our 89\% follow-up response rate is quite high given that the class was offered as free treatment in the clinic and no compensation was offered for follow-up responses. Nevertheless, it is plausible - perhaps likely - that only those who achieved best effects completed a follow-up PCS, thus introducing bias and again leading to an overestimation of FCR treatment effects. We used a common imputation method of last observation carried forward as an estimation of effects but we also acknowledge that this method may not be conservative enough. If we conservatively estimate that all participants who did not provide a follow-up PCS $(\mathrm{N}=6)$ had poor treatment response, then our finding for 51 FCR participants evidencing a clinically important improvement would represent a treatment responder rate of $67.1 \%$. As it is possible that our data were not missing entirely at random and, instead, may reflect an underlying difference between those who completed the PCS at various follow-ups and those who did not, we urge that the results of the current study be interpreted with this caution in mind. Regardless, we underscore again that it will be important for future studies to phenotype non-responders and modify the intervention to address their unmet needs.

The single-session FCR class appears to hold promise as an economical, high-impact, efficient, easily delivered, non-pharmacologic treatment for PC. Additional research is needed to clarify the role of depression, anxiety, and sex on treatment response, and to further tailor the intervention to meet the specific needs of subpopulations. Larger, controlled studies are needed to determine the durability of treatment effects, to characterize responders and non-responders, and the impact of treatment effects on pain and related outcomes. Ideally, future research may determine the efficacy of FCR in reducing suffering and reliance on less effective pharmacologic and interventional medical approaches for pain management across settings.

\section{Acknowledgments}

We acknowledge support from National Institutes of Health (NIH): National Center for Complementary and Alternative Medicine (NCCAM) P01AT006651 (SCM), P01AT006651S1 (SCM and BDD), K24DA029262 (SCM), HHSN271201200728P (SCM), UL1 RR025744 from National Center for Research Resources (NCRR) (Stanford University); and from the Chris Redlich Pain Research Endowment (SCM).

\section{Disclosure}

The authors report no conflicts of interest in this work.

\section{References}

1. Institute of Medicine (US) Commitee on Advancing Pain Research, Care, and Education, Relieving Pain in America: A Blueprint for Transforming Prevention, Care, Education, and Research. Washington (DC): National Academies Press (US); 2011.

2. IASP. Unrelieved Pain is a Major Global Healthcare Problem. Available from: http://www.iasp-pain.org/files/Content/ContentFolders/Globa 1YearAgainstPain2/20042005RighttoPainRelief/factsheet.pdf. 2011. Accessed March 24, 2014.

3. Sullivan MJ, Bishop SR, Pivik J. The Pain Catastrophizing Scale: Development and validation. Psychol Assess. 1995;7(4):524-532.

4. Martin CE, Johnson E, Wechter ME, Leserman J, Zolnoun DA. Catastrophizing: a predictor of persistent pain among women with endometriosis at 1 year. Hum Reprod. 2011;26(11):3078-3084.

5. Wertli MM, Burgstaller JM, Weiser S, Steurer J, Kofmehl R, Held U. Influence of catastrophizing on treatment outcome in patients with non-specific low back pain: a systematic review. Spine (Phila Pa 1976). 2014;39(3):263-273.

6. Vissers MM, Bussmann JB, Verhaar JA, Busschbach JJ, Bierma-Zeinstra SM, Reijman R. Psychological Factors Affecting the Outcome of Total Hip and Knee Arthroplasty: A Systematic Review. Semin Arthritis Rheum. 2012;41(4):576-588.

7. Litt MD, Porto FB. Determinants of pain treatment response and nonresponse: identification of TMD patient subgroups. J Pain. 2013;14(11):1502-1513.

8. Spinhoven P, Ter Kuile M, Kole-Snijders AM, Hutten Mansfeld M, Den OUden DJ, Vlaeyen JW. Catastrophizing and internal pain control as mediators of outcome in the multidisciplinary treatment of chronic low back pain. Eur J Pain. 2004;8(3):211-219.

9. Jensen MP, Turner JA, Romano JM. Changes in beliefs, catastrophizing, and coping are associated with improvement in multidisciplinary pain treatment. J Consult Clin Psychol. 2001;69(4):655-662.

10. Karels CH, Bierma-Zeinstra SM, Burdorf A, Verhagen AP, Nauta AP, Koes BW. Social and psychological factors influenced the course of arm, neck and shoulder complaints. J Clin Epidemiol. 2007;60(8):839-848.

11. Hill JC, Lewis M, Sim J, Hay EM, Dziedzic K. Predictors of poor outcome in patients with neck pain treated by physical therapy. Clin J Pain. 2007;23(8):683-690.

12. Granot M, Ferber SG. The roles of pain catastrophizing and anxiety in the prediction of postoperative pain intensity: a prospective study. Clin J Pain 2005;21(5):439-445.

13. Severeijns R, Vlaeyen JW, van den Hout MA, Weber WE. Pain catastrophizing predicts pain intensity, disability, and psychological distress independent of the level of physical impairment. Clin J Pain. 2001;17(2):165-172.

14. Sullivan MJ, Lynch ME, Clark AJ. Dimensions of catastrophic thinking associated with pain experience and disability in patients with neuropathic pain conditions. Pain. 2005;113(3):310-315.

15. Pinto PR, McIntyre T, Almeida A, Araujo-Soares V. The mediating role of pain catastrophizing in the relationship between presurgical anxiety and acute postsurgical pain after hysterectomy. Pain. 2012;153(1): 218-226.

16. Abbott AD, Tyni-Lenne R, Hedlund R. Leg pain and psychological variables predict outcome 2-3 years after lumbar fusion surgery. Eur Spine J. 2011;20(10):1626-1634.

17. Sommer M, et al. Predictors of acute postoperative pain after elective surgery. Clin J Pain. 2010;26(2):87-94.

18. Smeets RJ, van Geel AC, Kester AD, Knottnerus JA. Physical capacity tasks in chronic low back pain: what is the contributing role of cardiovascular capacity, pain and psychological factors? Disabil Rehabil. 2007;29(7):577-586.

19. Holroyd KA, Drew JB, Cottrell CK, Romanek KM, Heh V. Impaired functioning and quality of life in severe migraine: the role of catastrophizing and associated symptoms. Cephalalgia. 2007;27(10): 1156-1165.

20. Keefe FJ, Lefebvre JC, Egert JR, Affleck G, Sullivan MJ, Caldwell DS. The relationship of gender to pain, pain behavior, and disability in osteoarthritis patients: the role of catastrophizing. Pain. 2000;87(3): 325-334. 
21. Picavet HS, Vlaeyen JW, Schouten JS. Pain catastrophizing and kinesiophobia: predictors of chronic low back pain. Am J Epidemiol. 2002;156(11):1028-1034.

22. Jensen MK, Thomsen AB, Hojsted J. 10-year follow-up of chronic non-malignant pain patients: opioid use, health related quality of life and health care utilization. Eur J Pain. 2006;10(5):423-433.

23. Papaioannou M, et al. The role of catastrophizing in the prediction of postoperative pain. Pain Med. 2009;10(8):1452-1459.

24. Martel MO, Wasan AD, Jamison RN, Edwards RR. Catastrophic thinking and increased risk for prescription opioid misuse in patients with chronic pain. Drug Alcohol Depend. 2013;132(1-2):335-341.

25. Burton AK, Tillotson KM, Main CJ, Hollis S. Psychosocial predictors of outcome in acute and subchronic low back trouble. Spine. 1995;20(6):722-728.

26. Linton SJ. Do psychological factors increase the risk for back pain in the general population in both a cross-sectional and prospective analysis? Eur J Pain. 2005;9(4):355-361.

27. Burns JW, et al. Do changes in cognitive factors influence outcome following multidisciplinary treatment for chronic pain? A cross-lagged panel analysis. J Consult Clin Psychol. 2003;71(1): 81-91.

28. Campbell $\mathrm{CM}$, et al. Changes in pain catastrophizing predict later changes in fibromyalgia clinical and experimental pain report: crosslagged panel analyses of dispositional and situational catastrophizing. Arthritis Res Ther. 2012;14(5):R231.

29. Crombez G, et al. Fear-avoidance model of chronic pain: the next generation. Clin J Pain. 2012;28(6):475-483.

30. Eccleston C, Williams AC, Morley S. Psychological therapies for the management of chronic pain (excluding headache) in adults. Cochrane Database Syst Rev. 2012;11:CD007407.
31. Thorn BE, et al. A randomized clinical trial of targeted cognitive behavioral treatment to reduce catastrophizing in chronic headache sufferers. J Pain. 2007;8(12):938-949.

32. Osman A, et al. The Pain Catastrophizing Scale: further psychometric evaluation with adult samples. J Behav Med. 2000;23(4):351-365.

33. Osman A, et al. Factor structure, reliability, and validity of the Pain Catastrophizing Scale. J Behav Med. 1997;20(6):589-605.

34. Van Damme S, et al. A confirmatory factor analysis of the Pain Catastrophizing Scale: invariant factor structure across clinical and non-clinical populations. Pain. 2002;96(3):319-324.

35. Harris PA, et al. Research Electronic Data Capture (REDCap) A metadata-driven methodology and workflow process for providing translational research informatics support. J Biomed Inform. 2009;42(2):377-381.

36. Dworkin $\mathrm{RH}$, et al. Interpreting the clinical importance of treatment outcomes in chronic pain clinical trials: IMMPACT recommendations. J Pain. 2008;9:105-121.

37. Musil CM, Warner CB, Yobas PK, Jones SL. A comparison of imputation techniques for handling missing data. West J Nurs Res. 2002;24(7):815-829.

38. Theunissen M, Peters ML, Bruce J, Gramke HF, Marcus MA. Preoperative anxiety and catastrophizing: a systematic review and meta-analysis of the association with chronic postsurgical pain. Clin J Pain. 2012;28(9):819-841.

39. Buenaver LF, et al. Catastrophizing and pain-coping in young adults: associations with depressive symptoms and headache pain. J Pain. 2008;9(4):311-319.

40. Richardson EJ, et al. Depressive symptoms and pain evaluations among persons with chronic pain: catastrophizing, but not pain acceptance, shows significant effects. Pain. 2009;147(1-3):147-152.
Journal of Pain Research

\section{Publish your work in this journal}

The Journal of Pain Research is an international, peer-reviewed, open access, online journal that welcomes laboratory and clinical findings in the fields of pain research and the prevention and management of pain. Original research, reviews, symposium reports, hypothesis formation and commentaries are all considered for publication.

\section{Dovepress}

The manuscript management system is completely online and includes a very quick and fair peer-review system, which is all easy to use. Visit http://www.dovepress.com/testimonials.php to read real quotes from published authors. 